\title{
Solvent Reorganization in Electron and Ion Transfer Reactions near a Smooth Electrified Surface: a Molecular Dynamics Study
}

\author{
Christoph Hartnig ${ }^{\dagger}$ and Marc T. M. Koper* \\ Contribution from the Schuit Institute of Catalysis, Laboratory of Inorganic Chemistry and \\ Catalysis, Eindhoven University of Technology, 5600 MB Eindhoven, The Netherlands
}

Received April 7, 2003; E-mail: m.t.m.koper@tue.nl

\begin{abstract}
Molecular dynamics simulations of electron and ion transfer reactions near a smooth surface are presented, analyzing the effect of the geometrical constraint of the surface and the interfacial electric field on the relevant solvation properties of both a monovalent negative ion and a neutral atom. The simulations show that, from the solvation point of view, ion adsorption is an uphill process due to the need to shed off the ion's solvation shell and displace water from the surface. Atom adsorption, on the other hand, has only a small barrier, related to the molecularity of the solvent. Both the electrostatic interaction of the ion with the solvent and the ion's solvent reorganization energy (the relevant parameter in the Marcus electron transfer theory) decrease as the surface is approached, whereas these parameters are not sensitive to the distance from the surface for the atom. This is a consequence of the importance of long-range electrostatic interactions for ion solvation and the importance of short-range interactions for atom solvation. The electric field either attracts or repels an ion to or from the surface, but the field has no influence on the solvent reorganization energy. By including the quantum-mechanical electron transfer between the metal surface and the ion/atom in solution in the MD simulation by using a model Hamiltonian, we calculated two-dimensional free energy surfaces for ion adsorption allowing for partial charge transfer, based on a fully molecular picture of ion solvation near the surface.
\end{abstract}

\section{Introduction}

Electron transfer (ET) reactions are of fundamental importance in various areas of chemistry. ${ }^{1}$ In many instances, ET reactions take place near interfaces, one part of the interface being the solvent (water) and the other part being a more or less solid or rigid surface such as a metal, semiconductor, or a large biomolecule. Furthermore, all these interfaces feature a charge separation and hence an electric double layer with an associated electric field.

The theoretical framework with which heterogeneous redox reactions are usually analyzed is the well-known Marcus theory. ${ }^{2-4}$ In the Marcus theory, the energy of activation of an ET reaction is related to the reorganization of the solvent in the vicinity of the redox ions, so as to allow for a radiationless electron tunneling satisfying the Franck-Condon principle. The Marcus theory is a partly phenomenological theory, which predicts that the energy of solvent reorganization $\lambda$ associated with the homogenoeus ET reaction between redox species 1 and 2 depends only on their respective radii $a_{1}$ and $a_{2}$, their

$\dagger$ Present address: Forschungszentrum Jülich, Institute for Materials and Processes in Energy Systems (IWV-3), D-52425, Jülich, Germany. Email: c.hartnig@fz-juelich.de.

(1) For example, see: Jortner, J.; Bixon, M. Adv. Chem. Phys. 1999, 106/107.

(2) Marcus, R. A. J. Chem. Phys. 1956, 24, 966.

(3) Marcus, R. A. J. Chem. Phys. 1965, 43, 679.

(4) Marcus, R. A. Angew. Chem., Int. Ed. Engl. 1993, 32, 1111

9840 J. AM. CHEM. SOC. 2003, 125, 9840-9845 separation $r$, and the optical and static dielectric constants, $\epsilon_{\mathrm{opt}}$ and $\epsilon_{\mathrm{s}}$, of the solvent:

$$
\lambda=e_{0}^{2}\left(\frac{1}{2 a_{1}}+\frac{1}{2 a_{2}}-\frac{1}{r}\right)\left(\frac{1}{\epsilon_{\mathrm{opt}}}-\frac{1}{\epsilon_{\mathrm{s}}}\right)
$$

with $e_{0}$ as the elementary charge. At a metal electrode, a modification of Marcus' formula exists which takes into account the electrostatic image interaction between the transferred electron and the metal; that is,

$$
\lambda=e_{0}^{2}\left(\frac{1}{2 a}-\frac{1}{4 r}\right)\left(\frac{1}{\epsilon_{\mathrm{opt}}}-\frac{1}{\epsilon_{\mathrm{s}}}\right)
$$

where $r$ is now the distance of the redox species from the metal electrode. The relationship among the solvent reorganization energy $\lambda$, the free energy of the reaction $\Delta G$, and the activation energy $\Delta G_{\text {act }}$ of the ET reaction is then given by the well-known quadratic equation:

$$
\Delta G_{\mathrm{act}}=\frac{(\lambda+\Delta G)^{2}}{4 \lambda}
$$

Hence, a theory for $\lambda$ allows the calculation of the activation energy and ultimately the reaction rate.

The Marcus theory has found widespread application, in the interpretation of both homogeneous and heterogeneous electron 
transfer reactions. It seems, however, that the success of the Marcus theory lies mainly in its qualitative predictions of macroscopic rate data. On the molecular level, more detailed simulation studies have identified some of the shortcomings of the Marcus theory in correctly predicting numbers or even qualitative trends. ${ }^{5-26}$

We have recently presented extensive molecular dynamics (MD) simulations of solvent reorganization near ions in water in order to assess the quality of some of the main assumptions of the Marcus theory, including a test of quantitative correctness of the Marcus formulas. ${ }^{25}$ MD simulations allow one to investigate in molecular detail the influence of changes in the ion's characteristics such as size and charge, on the energy of solvent reorganization. In agreement with previous simulations (see, e.g., ref 14), our study brought to light two important effects not taken into account in the Marcus theory. First of all, due to ion-dipole interactions, the effective radius of the ion is strongly charge dependent. In addition, in water this charge dependence is not symmetric as a water molecule can approach closer to a negative ion than to a positive ion. This "electrostriction" effect may lead to a difference of a factor of 2 in the solvent reorganization energy (and hence many orders of magnitude in the ET rate) between a neutral and monovalent negative redox species, rendering the solvent response for a redox couple consisting of a neutral and singly charged negative species highly nonlinear. As we have shown elsewhere, the latter effect may have important consequences for the correct modeling of electrocatalytic reactions such as the oxygen reduction. ${ }^{26}$ Second, for more strongly charged ions (doubly or triply), dielectric saturation near the ion leads to a significant lowering of the solvent reorganization energy as compared to the neutral or singly charged species. ${ }^{14,25}$

So far, the majority of the microscopic simulations dealing with solvent reorganization in ET reactions have considered redox species in the bulk of the solution. The purpose of this paper is to study the effect of the geometrical constraint imposed by the interface, as well as the electric field in the double layer, on the solvent reorganization near a neutral and a singly charged species, herein typically modeled as a chlorine atom and chloride ion, extending similar simulations that have been done in the past by Xia and Berkowitz ${ }^{21}$ and Rose and Benjamin. ${ }^{23}$ The surface in our study will be modeled as a smooth wall having

(5) Warshel, A. J. Phys. Chem. 1982, 86, 2218.

(6) Hwang, J.-K.; Warschel, A. J. Am. Chem. Soc. 1987, 109, 715.

(7) King, G.; Warschel, A. J. Chem. Phys. 1990, 93, 8682.

(8) Kuharski, R. A.; Bader, J. S.; Chandler, D.; Sprik, M.; Klein, M. L.; Impey, R. W. J. Chem. Phys. 1988, 89, 3248.

(9) Bader, J. S.; Kuharski, R. A.; Chandler, D. J. Chem. Phys. 1990, 93, 230.

(10) Maroncelli, M.; Fleming, G. R. J. Chem. Phys. 1988, 89, 5044.

(11) Maroncelli, M. J. Chem. Phys. 1991, 94, 2084.

(12) Carter, E. A.; Hynes, J. T. J. Phys. Chem. 1989, 93, 2184

(13) Fonseca, T.; Landanyi, B. M.; Hynes, J. T. J. Phys. Chem. 1992, 96 4085

(14) Yelle, R. B.; Ichiye, T. J. Phys. Chem. B 1997, 101, 4127

(15) Straus, J. B.; Voth, G. A. J. Phys. Chem. 1993, 97, 7388.

(16) Straus, J. B.; Calhoun, A.; Voth, G. A. J. Chem. Phys. 1995, 102, 529

(17) Calhoun, A.; Voth, G. A. J. Phys. Chem. 1996, 100, 10746.

(18) Calhoun, A.; Voth, G. A. J. Electroanal. Chem. 1998, 450, 253

(19) Calhoun, A.; Koper, M. T. M.; Voth, G. A. J. Phys. Chem. B 1999, 103 3442 .

(20) Calhoun, A.; Koper, M. T. M.; Voth, G. A. Chem. Phys. Lett. 1999, 305, 94.

(21) Xia, X.; Berkowitz, M. L. Chem. Phys. Lett. 1994, 227, 561

(22) Rose, D. A.; Benjamin, I. J. Chem. Phys. 1993, 100, 3545.

(23) Rose, D. A.; Benjamin, I. Chem. Phys. Lett. 1995, 234, 209.

(24) Smith, B. B.; Halley, J. W. J. Chem. Phys. 1994, 101, 10915.

(25) Hartnig, C.; Koper, M. T. M. J. Chem. Phys. 2001, 115, 8540

(26) Hartnig, C.; Koper, M. T. M. J. Electroanal. Chem. 2002, 532, 165. a small attractive interaction with the water. These results are of interest not only in understanding the effect of the geometrical constraint and the electric field on the ET solvent reorganization parameters but also in their application to modeling ion transfer reactions at interfaces. These reactions were considered some years ago in a theory by Schmickler. ${ }^{27}$ It is relatively straightforward to combine Schmickler's model Hamiltonian for the quantum-mechanical interaction with the metal electrode with the molecular dynamics simulation, ${ }^{16}$ enabling the computation of free energy surfaces of ion transfer near a metal electrode with a fully molecular treatment of the role of the solvent. These simulations hence extend previous MD simulations of ions near a metal-solution interface ${ }^{28}$ by incorporating the roles of ion adsorption, solvent reorganization (in the Marcus sense), and electron transfer into one and the same simulation.

In a more general vein, we believe the simulation results to be presented below are the first of their kind in connecting, from a molecular point of view, the Marcus theory for electron transfer and the process of ion or atom adsorption at the metal/ electrolyte interface, in which essentially no assumptions are made apart from the model potentials chosen for the interactions between the species and the surface. We believe this presents a significant step forward in understanding the role of the solvent in complex reactions taking place at the solid-liquid interface, or even at the biomolecule-liquid interface, of importance in catalysis, corrosion, materials science, and biochemical energy transduction.

\section{Computational}

The investigated system contains 511 water molecules and one redox species in a rectangular box with two-dimensional periodicity. The third dimension is confined on one side by a model electrode surface and on the other side by a slightly repulsive potential in order to prevent water molecules from evaporating. The repulsive potential was adjusted such that the experimental density of $1.0 \mathrm{~g} / \mathrm{cm}^{3}$ in the center of the slab was achieved.

Solvent molecules are described by the rigid SPC/E water potential;29 ion-water and ion-ion interactions consist of Lennard-Jones and Coulomb terms and are taken from ref 30. The oxygen atoms of the solvent molecules and the solute interact with the model platinum surface by an empirical (12-6) Lennard-Jones potential of the form

$$
V(z)=4 \epsilon\left[\left(\frac{\sigma}{z-z_{0}}\right)^{12}-\left(\frac{\sigma}{z-z_{0}}\right)^{6}\right]
$$

where $z_{0}$ denotes the position of the wall and $z$ denote the actual position of the atom under consideration. The 12-6 Lennard-Jones potential was chosen for its computational efficiency. The values for $\epsilon, \sigma$, and $z_{0}\left(\epsilon=30.1 \mathrm{~kJ} / \mathrm{mol}, \sigma=2.0 \AA, z_{0}=14.0 \AA\right)$ were parametrized in such a way that the density distribution perpendicular to the surface, as shown in Figure 1, mimics the well-known density distribution in systems with atomically resolved metal/platinum surfaces. ${ }^{31,32}$

The effects of a charged electrode were simulated by applying a constant field $E_{0}$. An exponentially decaying electric field perpendicular

(27) (a) Schmickler, W. Chem. Phys. Lett. 1995, 237, 152. (b) Schmickler, W. Electrochim. Acta 1996, 41, 2329.

(28) For some recent work and a review, see: (a) Crozier, P. S.; Rowley, R. L. Henderson, D. J. Chem. Phys. 2001, 114, 7513. (b) Dimitrov, I.; Raev, N. D.; Semerdzhiev, K. I. Phys. Chem. Chem. Phys. 2001, 3, 448. (c) Spohr, E. Solid State Ionics 2002, 150, 1.

(29) Berendsen, H. J. C.; Grigera, J. R.; Straatsma, T. P. J. Phys. Chem. 1987, $91,6269$.

(30) Lee, S. H.; Rasaiah, J. C. J. Phys. Chem. 1996, 100, 1420.

(31) Spohr, E. Chem. Phys. 1990, 141, 87.

(32) Kohlmeyer, A.; Hartnig, C.; Spohr, E. J. Mol. Liq. 1998, 78, 233. 


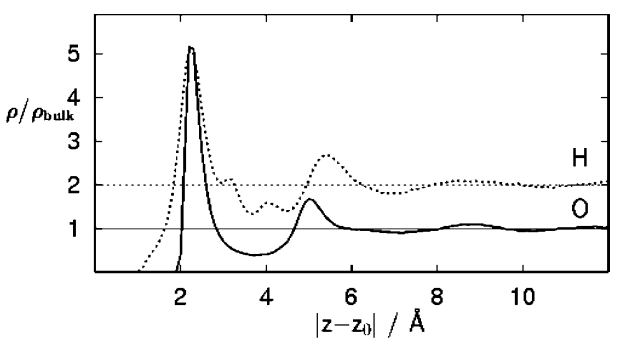

Figure 1. Hydrogen and oxygen density distributions as a function of the distance from the surface for our model wall-water potential eq 4.

to the metal surface was also studied, having the form

$$
E(z)=E_{0} \exp \left(-\frac{z-z_{0}}{l}\right)
$$

with $z_{0}$ as the origin of the field (position of the electrode, see above), $E_{0}$ as the electric field at $z_{0}$, and $l$ as the decay length, giving results identical to the constant field simulations. Hence, we show only results obtained with the constant field, where $E_{0}$ was chosen as either $-10^{9}$ or $+10^{9} \mathrm{~V} / \mathrm{m}$ for all field calculations.

Long-ranged electrostatic interactions were calculated using a 3DEwald summation with corrections for systems with slab geometry. ${ }^{33}$ The equations of motion were integrated using the Verlet algorithm with a time step of $1 \mathrm{fs}$. The constraints for the rigid water model were fulfilled using the SHAKE algorithm. A Nose-Hoover thermostat was used to keep the temperature constant at the desired value of 298.15 K.

The charge transfer between the metal electrode's electronic levels and the solute's orbital is modeled by an extended version of a Hamiltonian that is often referred to as the Anderson-NewnsHamiltonian. ${ }^{34-36}$ Briefly, the occupation of the acceptor orbital depends on the orientation of the solvent molecules, as monitored by the socalled generalized solvent-coordinate $\Delta E{ }^{37}$ the distance to the electrode $z$, and the electronic structure of the electrode. The average occupancy $n$ at any solvation state is given by

$$
n(\Delta E)=\frac{1}{2}+\frac{1}{\pi} \tan ^{-1}\left(\frac{\epsilon_{\mathrm{F}}-\epsilon_{\mathrm{a}}-\Delta E}{\Delta_{0} \exp \left(-b\left(z-z_{0}\right)\right)}\right)
$$

$n$ is the occupation number of the acceptor orbital, $\epsilon_{\mathrm{F}}$, the Fermi level of the metal, $\epsilon_{\mathrm{a}}$, the vacuum energy level of the acceptor's electron orbital, and $\Delta_{0} \exp \left(-b\left(z-z_{0}\right)\right)$, the distance dependent broadening of the energy orbital of the acceptor. This Hamiltonian was first used in the context of electron transfer reactions by Schmickler. ${ }^{38}$ The generalized solvent coordinate $\Delta E$ is defined as the electrostatic interaction energy between a negative test charge at the site of the solute and the prevailing configuration of solvent molecules and can be calculated straightforwardly during the MD simulation using:

$$
\Delta E=\sum_{j} \frac{-e_{0} q_{j}}{\left|r_{j}-R\right|}
$$

where $q_{j}$ is the charge of solvent atom $j$ (either hydrogen or oxygen), $r_{j}$ is the location of atom $j$ in the simulation cell, and $R$ is the location of the solute in the simulation cell. Further details on the implementation of the Anderson-Newns-Hamiltonian to molecular dynamics simulations of electron transfer reactions can be found in the paper by Straus et al.. ${ }^{16}$

(33) Yeh, I. C.; Berkowitz, M. L. J. Chem. Phys. 1999, 111, 3155.

(34) Anderson, P. W. Phys. Rev. 1961, 124, 41.

(35) Newns, D. M. Phys. Rev. 1969, 178, 1123.

(36) Muscat, J. P.; Newns, D. M. Prog. Surf. Sci. 1978, 9, 1.

(37) Marcus, R. A. Discuss. Faraday Soc. 1960, 29, 21.

(38) Schmickler, W. J. Electroanal. Chem. 1986, 204, 31.

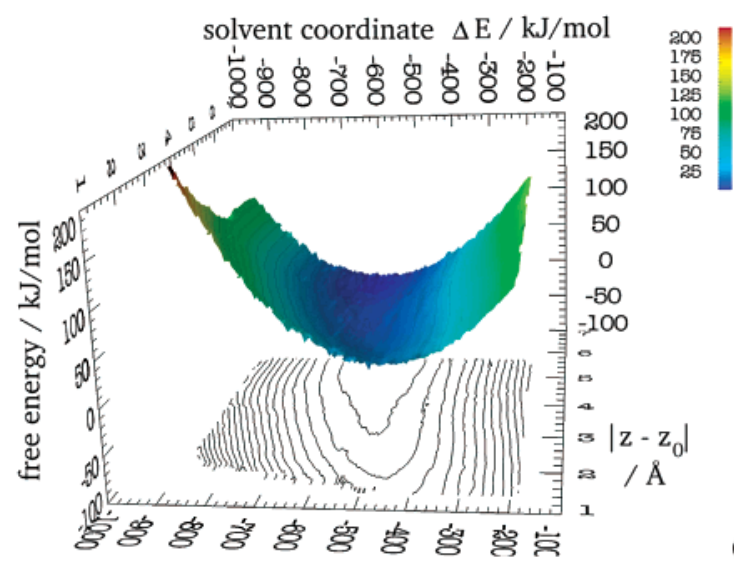

(a)

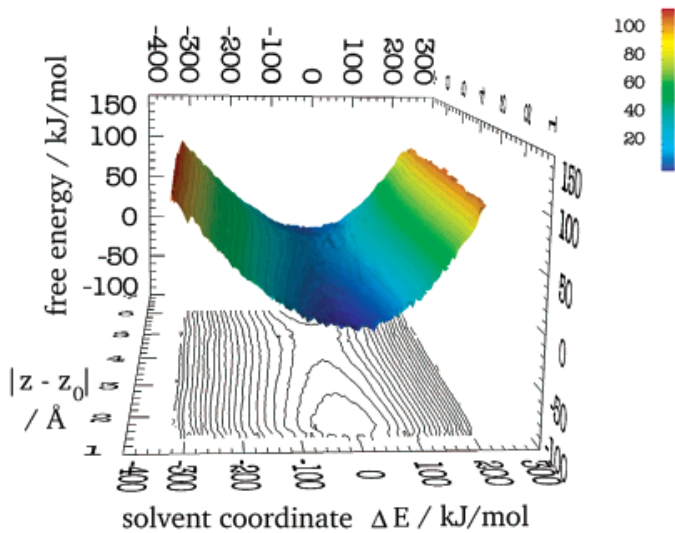

(b)

Figure 2. (a) Free energy surface for chloride as a function of the generalized solvent coordinate $\Delta E$ and the distance from the surface $z$. (b) Free energy surface for chlorine.

The dependence of the occupation number on the distance between the reactive species and the metal electrode as well as the solvent coordinate $\Delta E$ leads to a two-dimensional umbrella sampling along the two coordinates. The position of the reactive species was restrained at different distances by means of a parabolic umbrella potential. Details on the sampling procedure along the solvent coordinate can be found in our previous paper ${ }^{25}$ and references therein. The resulting windows were fitted together using a modified weighted-histogram analysis method.

\section{Results and Discussion}

Distance Dependence of Solvent Reorganization Energy. Figure 2 shows the two-dimensional free energy surfaces calculated by the procedures described in the previous section for the chloride ion (Figure 2a) and the chlorine atom (Figure $2 \mathrm{~b}$ ), as a function of the generalized solvent coordinate and the distance of the species from the wall. One can observe two important differences between the ion and the atom. First of all, as the ion approaches the surface, it has to overcome an energy penalty to become "contact adsorbed". This is clarified in Figure 3, which shows the "reaction path" for both the ion and the atom on their two-dimensional free energy surfaces. This "desolvation energy" is a result of the interactions of the ion with the solvent molecules: as the ion gets closer to the surface, it has to shed off part of its solvation shell and replace water molecules from the surface. Interestingly, there is a small barrier for the atom (dashed line in Figure 3), which occurs when the atom is at a distance from the surface for which it is 


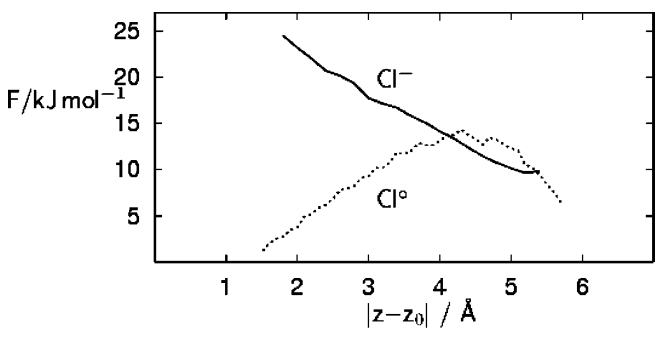

Figure 3. Reaction paths on the two-dimensional free energy surfaces of Figure 2 for $\mathrm{Cl}^{-}$(solid line) and $\mathrm{Cl}^{0}$ (dashed line).

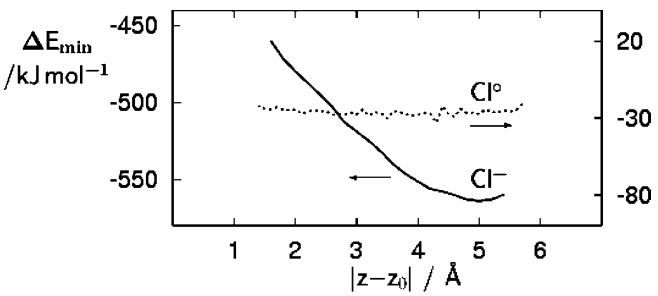

Figure 4. Minimum of the electrostatic energy of interaction between $\mathrm{Cl}^{-}$ (solid line) and $\mathrm{Cl}^{0}$ (dashed line) and the solvent as a function of the distance from the surface.

energetically unfavorable to fit a water molecule between the atom and the surface. However, there are no ion-dipole interactions, and from a solvation point-of-view, it is not as unfavorable for the atom to be contact adsorbed as it is for the ion. We note that the "desolvation energy" for the ion as calculated here is smaller than the values calculated in previous MD simulations of ions near surfaces. ${ }^{39-41}$ This is due to the fact that we have modeled the electrode surface as a smooth wall, with no corrugation potential for the water-surface interactions. This makes the water structure much more flexible and as a consequence leads to easier ion adsorption. This effect is also reflected in the fact that the self-diffusion coefficient of water parallel to a smooth wall is known to be higher than that next to a corrugated surface. ${ }^{42-44}$ Hence, we derive that a surface possessing a strong corrugation potential for water will generally possess a higher "desolvation energy" for ions than an energetically very smooth wall (this effect will be studied in more detail in a future publication ${ }^{45}$ ). Also note that, at a real metal surface, one would have to consider the attractive image interaction to the free energy surface shown in Figure 2a, making the actual event of ion adsorption a process with a barrier and a minimum in the contact-adsorbed state. ${ }^{27} \mathrm{In}$ the classical model considered here, the image interaction is simply an additive component to the overall free energy surface.

The decreasing interaction of the ion with the solvent as it approaches the surface can also be observed from a shift in the equilibrium value (i.e., where the free energy has a minimum) of the generalized solvent coordinate with decreasing distance from the surface. This is illustrated in Figure 4. Recall that the generalized solvent coordinate is the average electrostatic potential energy between the solvent and a test charge at the site of the ion, and since the ion is singly charged, it is the electrostatic part of the ion's solvation energy. It changes from

(39) Spohr, E. Chem. Phys. Lett. 1993, 207, 214.

(40) Pecina, O.; Schmickler, W.; Spohr, E. J. Electroanal. Chem. 1995, 394, 29.

(41) Spohr, E. Acta Chim. Scand. 1995, 49, 189.

(42) Sonnenschein, R.; Heinzinger, K. Chem. Phys. Lett. 1983, 102, 550

(43) Wallqvist, A. Chem. Phys. Lett. 1990, 165, 437.

(44) Lee, S. H.; Rossky, P. J. Chem. Phys. 1994, 100, 3334.

(45) Hartnig, C.; Koper, M. T. M., in preparation

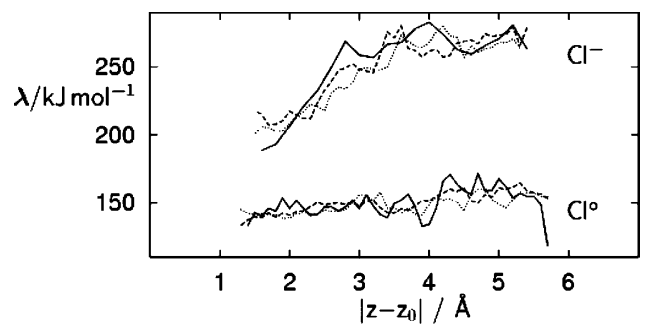

Figure 5. Solvent reorganization energy $\lambda$ as a function of the distance from the surface for $\mathrm{Cl}^{-}$and $\mathrm{Cl}^{0}$ for zero field (solid line), positive field $\left(10^{9} \mathrm{~V} / \mathrm{m}\right.$; dotted line $)$, and negative field $\left(-10^{9} \mathrm{~V} / \mathrm{m}\right.$; dashed line $)$.

ca. $-580 \mathrm{~kJ} / \mathrm{mol}$ far from the surface (in good agreement with Lee and Rasaiah ${ }^{30}$ ) to ca. $-460 \mathrm{~kJ} / \mathrm{mol}$ in the adsorbed state (solid line in Figure 4). No such effects are observed for the atom (see dashed line in Figure 4). Not surprisingly, this change in the average ion-solvent potential energy as the surface is approached is also reflected in the distance dependence of the solvent reorganization energy, which can be obtained from the curvature in the local minimum of the free energy surface at a certain fixed distance $z$ :

$$
\lambda(z)=\frac{1}{4}\left(\frac{\partial^{2} F(z)}{\partial \Delta E^{2}}\right)^{-1}
$$

The results are shown in Figure 5: the reorganization energy for the ion decreases as the ion approaches the surface, whereas that of the atom does not change, in harmony with the results shown in Figure 4 for the average ion- and atom-water electrostatic potential energies. Note that the electrostatic image term (see eq 2) is not included in this solvent reorganization energy. We emphasize that the effects discussed in this section are the result of a molecular description of solvation: a dielectric continuum model would not give the same results; in fact, such a model yields eq 2. Qualitatively, our results agree with earlier MD results of Xia and Berkowitz ${ }^{21}$ and Rose and Benjamin, ${ }^{23}$ who also observed that the location of the minimum of the generalized solvent coordinate and the solvent reorganization energy were smaller in the adsorbed state compared to the bulk state. Although Rose and Benjamin claimed only a small influence of the surface on the solvent reorganization energy, $\mathrm{Xia}$ and Berkowitz observed a decrease in the solvent reorganization energy of ca. $80-100 \mathrm{~kJ} / \mathrm{mol}$ for iodide and lithium ions in their adsorbed state compared to their bulk values. These numbers agree quite well with those extrapolated from Figure 5. However, the fact that this effect is absent for a neutral species was not mentioned in these two earlier studies. When taken together with the results of Xia and Berkowitz ${ }^{21}$ of smaller solvent reorganization energies for adsorbed iodide (a much larger ion than chloride) and lithium (a positive ion) compared to their respective bulk values, this lack of a distance dependence for the atom corroborates our deduction that the decrease in solvent reorganization energy of a charged species as it approaches the surface must reflect the decrease in long-range electrostatic interactions with the solvent. As only short-range solvation interactions are involved for neutral species, no significant changes are observed in their solvent reorganization energy as a function of the distance from the electrode surface.

The important result obtained here, namely that the dependence of the solvent reorganization energy on the distance from the surface is different for neutral and charged species, is not 


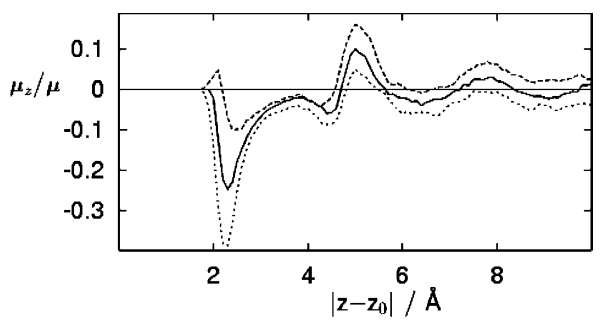

Figure 6. Water dipole density as a function of the distance from the surface for zero field (solid line), positive field $\left(10^{9} \mathrm{~V} / \mathrm{m}\right.$; dotted line), and negative field $\left(-10^{9} \mathrm{~V} / \mathrm{m}\right.$; dashed line).

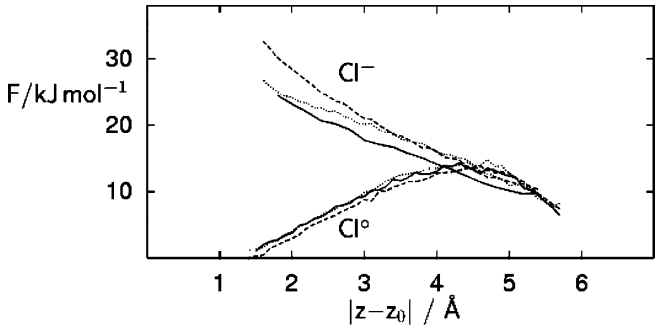

Figure 7. Reaction paths on the two-dimensional free energy surfaces for $\mathrm{Cl}^{-}$and $\mathrm{Cl}^{0}$ for zero field (solid line), positive field $\left(10^{9} \mathrm{~V} / \mathrm{m}\right.$; dotted line), and negative field $\left(-10^{9} \mathrm{~V} / \mathrm{m}\right.$; dashed line $)$.

incorporated in Schmickler's original model for ion transfer. ${ }^{27}$ In Schmickler's model, the distance dependence is qualitatively the same for all species, neutral and charged. In future work, ${ }^{45}$ we will study more generally the influence of the details of the water-surface potential and, in particular, its corrugation on this distance dependence of $\lambda$.

Field Dependence of the Free Energy and the Solvent Reorganization Energy. As mentioned in the Computational section, we have also studied the effect of an electric field on the free energy surfaces and their derived parameters, such as the solvent reorganization energy $\lambda$. The results obtained for a constant field and an exponentially decaying field are very similar, with the main difference that, for the exponentially decaying field, there is no effect observed far away from the surface. Figure 6 shows the average dipole moment density as function of the distance from the surface for negative, zero, and positive constant fields $\left(-1,0,1 \times 10^{9} \mathrm{~V} / \mathrm{m}\right.$, respectively), confirming the expected changes in the orientation of the water molecules.

Figure 7 displays the distance-dependent reaction paths for the chloride and chlorine species on their two-dimensional free energy surfaces. As expected, there is no effect of the field on the neutral species, the reaction paths being the same for all three fields. However, a clear influence on the reaction path for the chloride ion is observed. For the positive field (dotted line), the ion is attracted toward the surface, leading to a smaller slope of the reaction path compared to the field-free situation (solid line). For the negative field (dashed line), the ion is repelled from the surface, leading to a higher slope of the reaction path compared to the field-free situation. In strong contrast with the influence of the field on the free energy reaction path is the lack of influence of the field on the (distance dependent) solvent reorganization energy, for both the charged and neutral species (dashed and dotted lines in Figure 5). This lack of significant influence of the electric field on the electrontransfer parameters is in agreement with the earlier MD results of Rose and Benjamin. ${ }^{23}$ For the charged species, this can be understood in terms of the ion's proper electric field being

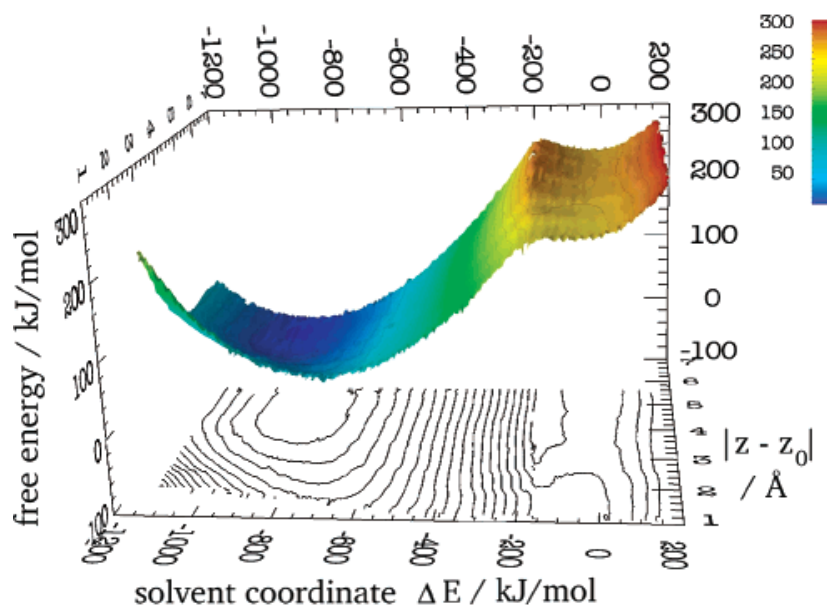

Figure 8. Free energy surface for the $\mathrm{F}^{-} / \mathrm{F}^{0}$ couple as a function of the generalized solvent coordinate $\Delta E$ and the distance from the surface $z$. The valley on the left corresponds to fluoride, and that on the right corresponds to fluorine.

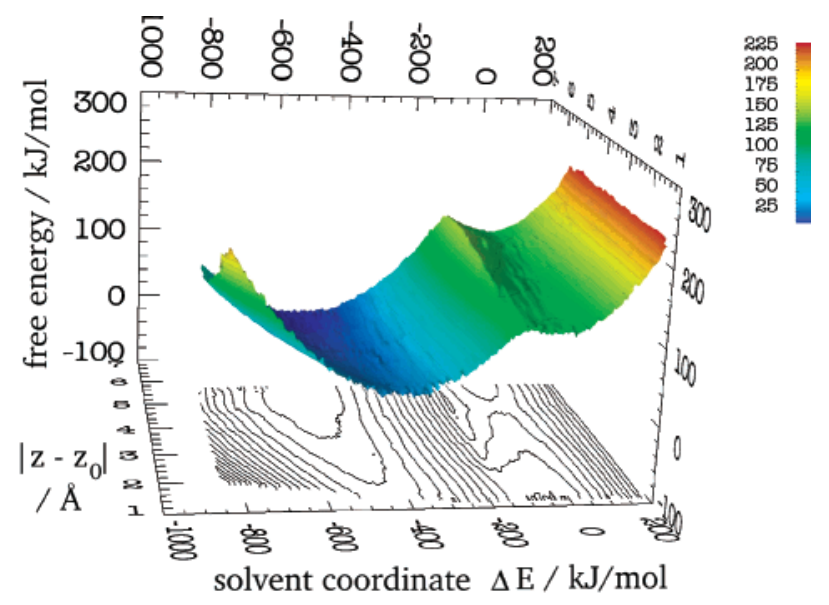

Figure 9. Free energy surface for the $\mathrm{Cl}^{-} / \mathrm{Cl}^{0}$ couple as a function of the generalized solvent coordinate $\Delta E$ and the distance from the surface $z$. The valley on the left corresponds to chloride, and that on the right corresponds to chlorine.

stronger than the surface electric field, such that the influence of the surface electric field can only be of second order. The electric field at the location of the first layer of water molecules around the chloride ion (taken at the maximum of the $\mathrm{Cl}-\mathrm{O}$ radial distribution function, ca. $3.1 \AA^{25}$ ) is indeed at least an order of magnitude higher than the electric double layer field applied in our simulations. One may expect an influence only for very (unrealistically) strong fields and/or large ions. However, the lack of influence of the field on the solvent reorganization energy of the neutral species is more surprising. Apparently, the applied field is not strong enough to alter the local solvent configurations such that the short-range interactions with the neutral species are significantly perturbed. Perhaps a more marked influence of the field may be expected for a neutral dipolar species, but such a system has not been studied yet.

Including Charge Transfer between Ion and Metal Electrode. Finally, we present in Figures 8 and 9 the free energy surfaces for fluoride and chloride adsorption on our model surface, taking into account the electronic interaction between the ion/atom and the (metal) electrode. Here, we have taken the electron affinities equal to their experimental values $\left(\epsilon_{\mathrm{a}}=\right.$ 3.40 and $3.614 \mathrm{eV}$ for fluorine and chlorine, respectively) and the Fermi level of the metal equal to the negative of the work 
function of platinum $\left(\epsilon_{\mathrm{F}}=-5.3 \mathrm{eV}\right)$. The values for $\Delta_{0}$ and $b$ were $2 \mathrm{eV}$ and $1 \AA^{-1}$, as suggested approximately by ab initio quantum-chemical calculations. ${ }^{46}$

In these figures, one recognizes the ion and atom channels far from the surface, the ion channel being of considerably lower energy due to the electron affinity of the atom and the stabilization provided by the solvation energy. The appearance of these ion and atom channels in these Anderson-Newns (MD) simulations is well-known (see, e.g., refs $15-18,27$, and 37) and is related to the fact that the Hamiltonian favors states with an integral charge far from the surface, that is, where the electronic interaction is small. Closer to the surface, however, this distinction between ion and atom channels disappears as the free energy surface displays only one minimum as a function of the solvent coordinate due to the strong interaction with the electrode [large value of $\Delta=\Delta_{0} \exp \left(-b\left(z-z_{0}\right)\right)$ ], this effect being more pronounced for fluoride than for chloride. This minimum corresponds to the adsorbed state of the ion. The charge in the adsorbed state is no longer exactly -1 due to partial charge transfer. For fluoride, the simulation gives $q \approx$ - 0.97, whereas, for chloride, the minimum occurs for $q \approx$ -0.94 . This agrees with the expectation that chloride should discharge more in its adsorbed state than fluoride. The figures illustrate that for ion transfer processes, the reaction mainly proceeds along the reaction coordinate representing the distance from the surface and not along the generalized solvent coordinate, in agreement with Schmickler's model for ion transfer. ${ }^{27}$ From these surfaces, one may estimate rate constants for ion transfer by applying classical reaction rate theories, ${ }^{47}$ whereas dynamical aspects may also be studied by MD simulations, as has been done for electrochemical ion transfer processes without charge transfer by Pecina and Schmickler. ${ }^{48}$

The "desolvation energy" for the ion to become contact adsorbed is higher for fluoride than for chloride, as expected and observed experimentally. Fluoride has a stronger interaction with the solvent due its smaller size. However, we would like to point out once more that these desolvation energies are small due to the lack of corrugation of the surface-water potential.

The distance dependence of the solvent reorganization energy as obtained from the curvature of the atom and ion channels is not significantly different from the values obtained for the simulation without charge transfer, as the charge transfer to the ion and atom states is still relatively small. Hence, Figure 4 applies to the charge transfer model as well.

(46) Koper, M. T. M.; van Santen, R. A. Surf. Sci. 1998, 422, 118.

(47) Koper, M. T. M.; Schmickler, W. Chem. Phys. 1996, 211, 123.

(48) Pecina, O.; Schmickler, W. J. Electroanal. Chem. 1998, 450, 303.

\section{Conclusion}

In this paper, we have modeled electron transfer reactions near a charged surface and analyzed the effect of the geometrical constraint and the interfacial electric field on the relevant solvation properties of both monovalent negative ion and a neutral atom. To this end, two-dimensional free energy surfaces as a function of the generalized solvent coordinate and the distance from the surface were obtained from extensive molecular dynamics simulations using two-dimensional umbrella sampling.

From the solvation point of view (not taking into account image and chemical interactions of the ion with the metal surface), ion adsorption is an uphill process due to the need to shed off the ion's solvation shell and displace water from the surface. Atom adsorption, on the other hand, has only a small barrier, related to unfavorable configurations of the atom, surface, and solvent in which the separation between the atom and the surface is such that placing a water molecule between takes a relatively high energy. Both the electrostatic interaction of the ion with the solvent and the ion's solvent reorganization energy $\lambda$ (obtained from the curvature of the free energy surface by eq 8 ) decrease as the surface is approached, whereas these parameters are not sensitive to the distance from the surface for the atom. This is a consequence of the importance of longrange electrostatic interactions for ion solvation and the importance of short-range interactions for atom solvation. The electric field either attracts or repels an ion to or from the surface, but the field has no influence on the solvent reorganization energy. Finally, by including the quantum-mechanical electron transfer between the metal surface and the ion/atom in solution in the MD simulation by using a model Hamiltonian, we calculated two-dimensional free energy surfaces for ion adsorption allowing for partial charge transfer. As a result of the electronic interactions, close to the surface the barrier between the ion and the atom disappears and only one (adsorbed) state can be identified. These surfaces are based on a fully molecular picture of ion solvation near the surface and, as such, provide the molecular extension of the Marcus and Schmickler theories of electron and ion transfer at metal electrodes.

Acknowledgment. This research was supported by the Netherlands Organization for Scientific Research (NWO) and by a generous grant of supercomputer facilities by the Netherlands National Computing Facilities Foundation (NCF).

JA035498U 